\title{
Segundo Diplomado en Arqueometría (IIA-UNAM): una visión de conjunto entre las ciencias naturales y sociales
}

\author{
Óscar Hugo Jiménez Salas
}

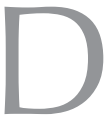

el 15 de agosto al 5 de diciembre de 2011 se llevó a cabo el segundo Diplomado en Arqueometría en el Instituto de Investigaciones Antropológicas de la Universidad Nacional Autónoma de México (IIAUNAM). Este importante acto académico, cuya coordinación general estuvo a cargo de los doctores Luis Barba Pingarrón e Isabel Villaseñor Alonso, se desarrolló en nueve módulos, constituidos por casi todas las disciplinas y ciencias que intervienen en las investigaciones arqueométricas en México y el extranjero. Puesto que éstas se sitúan en la frontera frecuentemente difusa entre las ciencias naturales y las sociales, formando una interfaz multidisciplinar en la que se aplican técnicas científicas para obtener información arqueológica, histórica y natural relevante generada en contextos espaciales y temporales particulares - aquellos en que las sociedades antiguas llevaban a cabo actividades cotidianas y rituales-, el objeto principal del diplomado consistió en aportar una visión de conjunto que demostrara que, con base en la práctica de una y otra de las ciencias antedichas, es factible construir puentes de comunicación antropológica entre los contextos culturales y materiales que se producen durante las investigaciones arqueológicas y de diagnóstico, conservación y restauración del patrimonio cultural en nuestro país (Figura 1).

\section{Actualización teórica y metodológica}

La diversidad temática abundó a lo largo de las 124 horas del diplomado, dividido en nueve módulos: 1) Introducción a la arqueometría, 2) Prospección arqueológica, 3) Datación, 4) Estudio y caracterización de materiales culturales, 5) Química de superficie de ocupación y análisis de residuos en materiales porosos, 6) Arqueología ambiental y estudio de restos óseos, 7) Análisis espaciales y simulación, 8) Aplicaciones en conservación y restauración, y 9) La perspectiva interdisciplinaria en la arqueometría y reflexiones finales, los cuales fueron coordinados por los doctores Luis Barba (1 y 2), Laura Beramendi (3) y José Luis Ruvalcaba (4); por el maestro Agustín Ortiz (5), y los doctores Emily McClung y Abigail Meza (6), Jorge Herrera (7 y 9), Valerie Magar (8) e Isabel Villaseñor (1, 8 y 9). Cada módulo comprendió, a su vez, varios temas particulares, tratados ampliamente por especialistas de renombre en el medio 
académico nacional e internacional, cuyo aporte se centró en ejemplos concretos de su trabajo realizado durante varios años de investigación, lo que contribuyó a contextualizar los aspectos teóricos presentados;

\section{Diplomado en Arqueometría}

Coordinadores generales: Dr. Luis Barba Pingarrón y Dra. Isabel Villaseñor Alonso

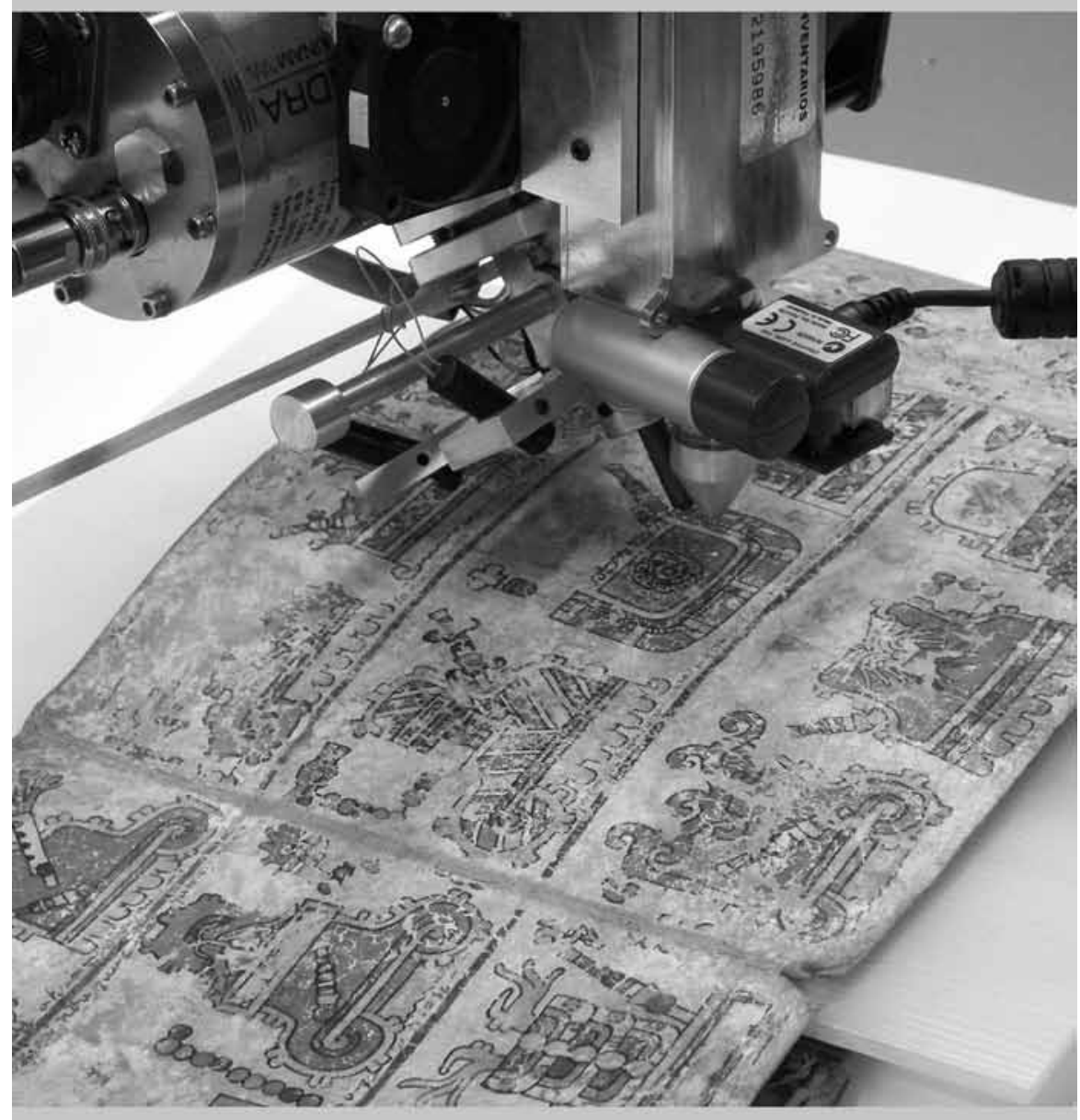

\section{Prospección arqueológica • Caracterización de materiales •} Datación • Química de superficies de ocupación • Análisis espaciales y simulación - Arqueología ambiental y estudios de restos óseos • Conservación y restauración

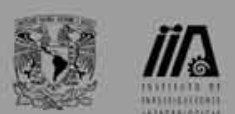

Costo: $\$ 7.000$, diferido en 3 pagos. $50 \%$ descuento a estudiantes UNAM, 35\% a estudiantes de otras entidades. Dirigido a pasantes y profesionistas de ciencias experimentales, arqueologia, conservación-restauración y disciplinas afines. Agosto - diciembre 2011. Lunes y jueves 4:00-8:00 pm, Instituto de Investigaciones Antropológicas, UNAM. Minimo 15 alumnos para la apertura del diplomado. Informes: diplomado.arqueometria@gmail.com

FIGURA 1. Cartel conmemorativo (Cortesía: Isabel Villaseñor Alonso, 2011).

ron dispuestos, ya en sus conferencias como fuera de éstas, a transmitir sus experiencias con una actitud pedagógica sobresaliente, así como a trazar constantemente pautas para la discusión y la crítica de posiciones, muchas veces encontradas, entre los participantes del diplomado y cada uno de los propios especialistas. Este hecho fue significativo por razón de que permitió reflexionar acerca de posturas filosóficas regularmente no incluidas en los trabajos de investigación arqueométrica, demostró que los temas deben criticarse y abordarse de acuerdo con preguntas previamente seleccionadas, además hizo hincapié en la necesidad de que un mayor número de estudiantes y estudiosos se dediquen de tiempo completo a este tipo tan variado de investigaciones.

Tanto la riqueza de los temas como la vasta perspectiva de sus planteamientos dieron lugar, asimismo, a considerar la toma de una posición disciplinar ante los diferentes ámbitos de competencia dentro de la conservación, la protección, la restauración y la investigación científica del patrimonio cultural mexicano. Es decir, la cantidad tan grande de disciplinas y subdisciplinas existentes, y de otras que empiezan a tomar cuerpo, implica que, por la imposibilidad de consagrarse a todas ellas, es indispensable hacer una elección que dependerá del conocimiento e intuición del interesado, así como de su eventual adhesión u oposición a una determinada corriente del pensamiento contemporáneo.

Por otro lado, la visita a laboratorios especializados: de termoluminiscencia, del acelerador Pelletron, de osteología, de etnobotánica, de radiocarbono, entre varios más, y la explicación del funcionamiento de equipos y metodologías avanzadas resaltaron la incuestionable necesidad de utilizar nuevas técnicas instrumentales dentro de las ciencias, ya sean de los materiales, de la vida, de la tierra o, desde luego, de las sociales. Sin embargo, en un devenir histórico difícil de aprehender, 


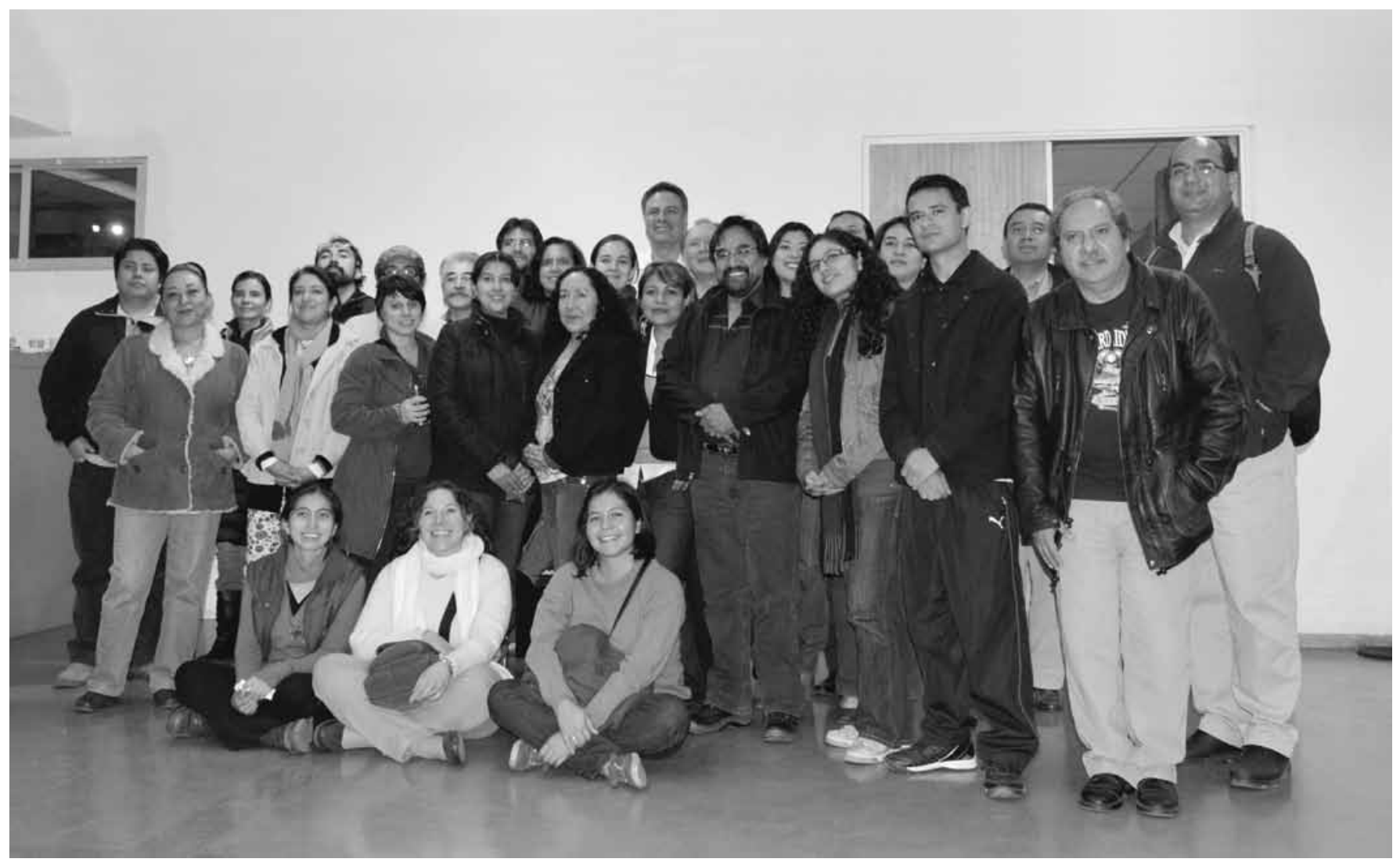

FIGURA 2. Expositores y asistentes al evento (Cortesía: Manuel Espinosa, 2011).

no todo es tecnología, sino también reflexión teórica, particularmente cuando se combinan trabajos de gabinete, de campo y de laboratorio. En consecuencia, la arqueometría se proyectó como una actividad científica consolidada, donde las investigaciones propias de cada disciplina se vinculan y coadyuvan a dar respuesta a la misma pregunta de tipo social, incluso con aquellas que aspiran a sobrepasar sus propias fronteras.

De esta manera, en un par de sesiones finales, en las que expusieron un estudio de caso sobre el Templo Mayor y la perspectiva de la arqueometría en México y América Latina, impartidas por los doctores Leonardo López Luján y Luis Barba Pingarrón, respectivamente, se dejó constancia tanto de la construcción de conocimiento científico específico como del potencial que ya existe en México para seguir realizando investigación arqueométrica. Ambos estudios abrieron ventanas en cuanto a considerar análisis espaciales detallados, temáticas nuevas y aspectos teóricos de cada disciplina, además de reflexionar acerca de asuntos epistemológicos, tecnocráticos y de divulgación científica, y propiciar mayor número de actos públicos para dar a conocer los resultados alcanzados, y con ello acrecentar el interés por este tipo de investigaciones (Figura 2).

\section{Conclusiones}

La investigación en arqueometría esclarece y rescata una visión de conjunto dentro de los estudios antropológicos en México, a la par que evita considerar la investigación arqueológica como una actividad que practica procedimientos metodológicos correspondientes a las ciencias naturales. Durante el diplomado que es objeto de esta reseña se puso en evidencia la importancia no sólo de propiciar la alianza y la retroalimentación permanentes entre instituciones sino de compartir datos y de evitar el aislamiento originado por la especialización. Para lograr lo anterior todavía falta, como manifestó el doctor Barba Pingarrón, una mayor comunicación entre especialistas, la creación de bases de datos accesibles a todos y el impulso a la creación de publicaciones donde se divulguen los resultados obtenidos, todo ello para estimular una reflexión teórico-metodológica que produzca mayor conocimiento arqueométrico.

Ciertamente, durante el diplomado quedó pendiente la realización de una sesión que versara sobre los enfoques teóricos en arqueología y la adopción de una posición teórico-metodológica para la realización de investigaciones arqueológicas, lo cual se debió, por un lado, a que el énfasis del diplomado se puso en las disciplinas de las ciencias naturales $y$, por el otro, a que los participantes procedían principalmente de una preparación en arqueología y sabían de dichos enfoques, mientras quienes 
venían del ámbito de las ciencias naturales los desconocían. Esta referencia común hubiera ayudado a tener una mirada más clara del presente ante el estudio del pasado. Adicionalmente, en relación con los conferencistas vale la pena señalar que algunos de ellos no aportaron el material gráfico mostrado a los participantes, situación que contradice la idea de colaborar, compartir y discutir la información.

Para finalizar, un comentario sobre la intención de impartir un tercer diplomado, en esa ocasión con la modalidad a distancia; es decir, mediante la utilización de las nuevas tecnologías de comunicación e información. Lo deseable es que sea pronto, pues contribuiría con lo arriba señalado: a fomentar la vinculación entre instituciones y a compartir experiencias metodológicas que redunden en más y mejores publicaciones sobre las investigaciones arqueométricas. ${ }^{1}$

\footnotetext{
${ }^{1}$ Respecto de éstas ha de señalarse, que, debido a que los estudios arqueométricos no son de exclusiva utilidad para la arqueología, existe una gama muy amplia de revistas periódicas, en distintos idiomas, fácilmente consultables. Algunas consideradas como las de mayor tradición son: Archaeometry, ArchéoSciences (revue d'archéométrie), American Antiquity, Radiocarbon, Geoarchaeology y, más recientemente, Archaeological and Anthropological Sciences. Asimismo, para una
}

\section{Agradecimientos}

Intervención agradece a los doctores Isabel Villaseñor y Manuel Espinosa por el material gráfico que ilustra esta reseña.

\footnotetext{
revisión histórica de la arqueometría posterior a la realización del trigésimo segundo simposio internacional realizado en la ciudad de México del 15 al 19 de mayo de 2000, editado por el IIA-UNAM, véase Ignacio Montero Ruiz, Manuel García Heras y Elías López-Romero 2007, "Arqueometría: cambios y tendencias actuales", Trabajos de Prehistoria 64 (1):2340, documento electrónico disponible en [http://digital.csic.es/bitstream/10261/8681/1/ ARQUEOMETR\%C3\%8DA.pdf],consultado en julio de 2013.
}

\section{Resumen}

Reseña crítica sobre los contenidos presentados en el Segundo Diplomado en Arqueometría Ilevado a cabo del 15 de agosto al 5 de diciembre de 2011, en el Instituto de Investigaciones Antropológicas de la Universidad Nacional Autónoma de México (IIA-UNAM), evento que reunió a especialistas de México y el extranjero en torno a los avances, estudios y aplicación de las investigaciones arqueométricas.

\section{Palabras clave}

Arqueometría; Segundo Diplomado en Arqueometría; IIA; investigación

\section{Abstract}

Critical review on the contents presented at the Second Diploma Course in Archaeometry held from $15^{\text {th }}$ of $\mathrm{Au}$ gust to $5^{\text {th }}$ of December 2011 in the Instituto de Investigaciones Antropológicas, Universidad Nacional Autónoma de México (IIA-UNAM, Institute for Anthropological Research at the National Autonomous University of Mexico), an event which brought together specialists from Mexico and abroad to discuss the progress, studies and application of archaeometrical research.

\section{Key words}

Archaeometry; Second Diploma Course in Archaeometry; IIA; research

Título en inglés: Second Diploma Course in Archaeometry (IIA-UNAM): A Joint

Vision between the Natural and Social Sciences 